\title{
COMPORTAMIENTO PRODUCTIVO DEL PASTO OVILLO (Dactylis glomerata L.) EN RESPUESTA AL PASTOREO ${ }^{1}$
}

\author{
Filogonio Jesús Hernández-Guzmán², Alfonso Hernández-Garay ${ }^{3}$,Eusebio Ortega-Jiménez, \\ Javier Francisco Enríquez-Quiroz ${ }^{5}$, Mauricio Velázquez-Martínez ${ }^{2}$
}

\begin{abstract}
RESUMEN
Comportamiento productivo del pasto ovillo (Dactylis glomerata L.) en respuesta al pastoreo. El objetivo del estudio fue determinar los patrones de variación en los componentes de la productividad forrajera del pasto ovillo (Dactylis glomerata L.) bajo pastoreo. En Montecillo, Texcoco, Estado de México, México, de junio 2005 a junio 2006, se estudió la combinación de tres frecuencias (21,28 y 35 días) y dos intensidades $(5-7$ y $9-11 \mathrm{~cm}$ ) en el forraje residual de pastoreo, en un diseño de bloques al azar con tres repeticiones. Se evaluó el rendimiento anual y la distribución estacional de forraje, tasa de crecimiento, composición botánica y morfológica y densidad de tallos. La frecuencia de pastoreo de 35 días superó a las de 21 y $28(\mathrm{P}<0,05)$ en el rendimiento anual, durante primavera y verano. Independientemente de la frecuencia e intensidad de pastoreo, el rendimiento estacional se distribuyó de la siguiente forma: 49,5, 31,6, 14,1 y 4,8\% para primavera, verano, invierno y otoño, respectivamente. La mayor tasa de crecimiento se registró en primavera al pastorear cada 35 días y la menor en otoño al pastorear cada 28 días $(\mathrm{P}<0,01)$. Durante invierno se dio mayor presencia de otras especies, siendo el pastoreo cada veintiún días a una intensidad ligera el que originó un aporte al rendimiento de otras especies del $60 \%$. No se presentaron efectos de intensidad ni de frecuencia de pastoreo en la densidad de tallos. El mayor rendimiento y tasa de crecimiento se obtuvieron al pastorear cada 35 días.
\end{abstract}

Palabras clave: manejo del pastoreo, acumulación de forraje, distribución estacional.

\begin{abstract}
Productive behaviour of orchard grass (Dactylis glomerata L.) to sheep grazing management. The aim of this study was to determine the yield variation patterns in orchard grass (Dactylis glomerata L.) under grazing management. This study was performed in Montecillo, Texcoco state, Mexico from June 2005 to July 2006. Three grazing frequencies (21, 28 and 35 days) and two grazing intensities (5-7 and 9-11 cm residual sward high) were evaluated in a completely random block design with three replicates. The variables grass yield, seasonal distribution, growth rate, botanical and morphological composition and tiller population density were all evaluated. A 35 day-grazing frequency produced the highest yield $(\mathrm{P}<0.05)$. Independently of the grazing intensity and its frequency the seasonal yield was: $49.5,31.6,14.1$ and $4.8 \%$ for spring, summer, winter and fall, respectively. The highest growth rate was registered during spring when grazing occurred every 35 days, and the lowest during autumn when grazing occurred every 28 days $(\mathrm{P}<0.01)$. During winter grazing only occurred every 21 days, and this may have led to $60 \%$ contribution to total yield by other species. Results also suggest that tiller density was not affected by grazing intensity and frequency on tiller population density.
\end{abstract}

Keywords: grazing management, grass yield, seasonal distribution.

1 Recibido: 5 de junio, 2014. Aceptado: 22 de setiembre, 2014. Trabajo forma parte de Tesis de Maestría en Ciencias Agrícolas del primer autor. Colegio de Postgraduados Campus Montecillo, Texcoco, estado de México.

2 Instituto Nacional de Investigaciones Forestales, Agrícolas y Pecuarias, Región Noreste, Campos Experimental San Luis. Carr. San Luis Matehuala km. 14.5, Soledad de Graciano Sánchez, estado de San Luis Potosí, México. Teléfono: 01 (444) 852 4316. Código Postal 78430. hernandez.filogonio@inifap.gob.mx, velazquez.mauricio@inifap.gob.mx

3 Colegio de Postgraduados Campus Montecillo. Carretera México-Texcoco km 34.5 Montecillo, municipio de Texcoco, Estado de México. Código Postal 56230. Tel 52 (595) 9520200 ext 1711 y 1716. hernan@colpos.mx (Autor para correspondencia).

4 Colegio de Postgraduados Campus Veracruz. Km. 88.5 Carretera Xalapa - Veracruz, Predio Tepetates entre Puente Jula y Paso San Juan, Código Postal. 91690. Apartado Postal 421. Veracruz, Ver., México. 52 (229) 2010770 Ext. 64301, 64302, 64307. eortegaj@colpos.mx

5 Instituto Nacional de Investigaciones Forestales, Agrícolas y Pecuarias, Campo Experimental La Posta, Región Golfo Centro. Carretera Veracruz-Córdoba km 22.5, Paso del Toro Medellín de Bravo, Estado de Veracruz, México. Código Postal 94277.52 (229) 2622222 y 52 (229) 26 22223. enriquez.javier@inifap.gob.mx

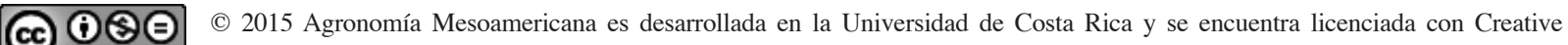
EY No No Commons Reconocimiento-NoComercial-SinObraDerivada 3.0 Costa Rica. Para más información escríbanos a pccmca@ucr.ac.cr 


\section{INTRODUCCIÓN}

El pasto ovillo (Dactylis glomerata L.) es una gramínea de uso común en praderas de clima templado de México. Es una especie con gran capacidad de crecimiento en primavera $(3500 \mathrm{~kg} \mathrm{MS} / \mathrm{ha}$ a las cuatro semanas de rebrote) y verano $(2500 \mathrm{~kg}$ a las cuatro semanas de rebrote) (Velasco et al., 2001), con resistencia moderada al frío y sequía, además de tener una buena calidad nutritiva (20\% proteína y 65\% de DIVMS (Kemp et al., 1999; Villareal et al., 2014). La frecuencia e intensidad de pastoreo, determinan la tasa de crecimiento del cultivo, materia seca acumulada, composición botánica, persistencia, densidad y peso de los tallos que integran la pradera (Parsons y Penning, 1988; Hill, 1989; Richards, 1993) por lo que es importante estudiar el efecto de estos factores de manejo en los ambientes particulares de explotación forrajera.

Una frecuencia e intensidad de pastoreo adecuadas, disminuyen las pérdidas por senescencia y descomposición del forraje e incrementan el crecimiento neto de la pradera (Matthew et al., 1999; Hernández-Garay et al., 2000); además permiten que esta se recupere más rápidamente de la defoliación (Da Silva y Hernández Garay, 2010). Los pastoreos severos y frecuentes reducen el rendimiento y persistencia de las especies forrajeras, mientras que con pastoreos menos frecuentes el rendimiento puede ser mayor, pero de menor calidad (Velasco et al., 2007). El impacto negativo de los pastoreos severos sobre el rendimiento y persistencia de las praderas, se ha explicado por la remoción de los meristemos de crecimiento (Anderson et al., 1989), la menor área foliar remanente y el agotamiento de los carbohidratos de reserva (Hodgson, 1990; Turner et al., 2006).

La persistencia y el rendimiento de forraje de una pradera están determinados por el efecto combinado del patrón estacional de aparición y muerte de tallos, por lo que la productividad de una especie depende del mantenimiento de un balance entre la densidad y peso de los tallos, balance que es afectado especialmente por la frecuencia e intensidad de cosecha (Hodgson, 1996; Hernández-Garay et al., 1999). Algunos estudios (Hernández-Garay et al., 1997a) han demostrado que se puede incrementar el rendimiento de forraje debido al aumento en la densidad y el peso de tallos. Aunque en pastos como el ballico perenne cv. 'Ruanui' (Lolium multiflorum L.), se ha observado que los nuevos tallos provienen de tallos sin florecer y son formados a una tasa constante durante el año (Matthew et al., 1995). En la misma especie, en Montecillo Edo. de México, Velasco et al. (2007) encontraron que la mayor acumulación de biomasa de hojas por unidad de superficie, ocurrió con cortes cada cuatro semanas, en comparación con los de cada dos y seis semanas, porque la mejor frecuencia combinó una densidad relativamente alta de tallos con un tamaño intermedio de los mismos, lo que permitió obtener la máxima tasa neta de aparición de nuevos tallos (tasa de aparición tasa de muerte) y, por tanto, la mayor cantidad de hojas por unidad de superficie.

En México es escasa la información científica sobre la dinámica de crecimiento del pasto ovillo al variar la frecuencia e intensidad del pastoreo, por tanto el objetivo del estudio fue determinar los patrones de variación en los componentes de la productividad forrajera del pasto ovillo (Dactylis glomerata L.) bajo pastoreo.

\section{MATERIALES Y MÉTODOS}

\section{Sitio experimental}

El experimento se llevó a cabo de junio de 2005 a junio de 2006, en una pradera de pasto ovillo (Dactylis glomerata L.) variedad Potomac, ubicada en Montecillo, Texcoco, Estado de México a 2240 msnm, $19^{\circ} 31^{\prime} \mathrm{LN}$ y $98^{\circ} 53^{\prime} \mathrm{LO}$. El suelo del área

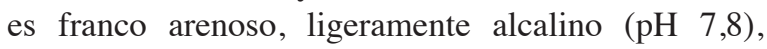
con $2,4 \%$ de materia orgánica y está clasificado como un Typic ustipsamments (Ortiz, 1997). El clima es templado subhúmedo, con lluvias en verano, precipitación y temperatura media anual de $645 \mathrm{~mm}$ y $15^{\circ} \mathrm{C}$, respectivamente (García, 1988). La temperatura promedio mensual más baja $\left(11,6^{\circ} \mathrm{C}\right)$ y más alta $(18,4$ $\left.{ }^{\circ} \mathrm{C}\right)$ se registran en enero y mayo, respectivamente.

\section{Manejo de la pradera y tratamientos}

La pradera tenía dos años de sembrada al iniciar el experimento y presentaba $80 \%$ de pasto ovillo. En la época seca (octubre a mayo) se regó por aspersión cada quince días hasta capacidad de campo, y no se fertilizó. El 16 de julio, previo a la aplicación de los tratamientos, toda la pradera fue pastoreada a una 
altura de $8 \mathrm{~cm}$, para ello, la pradera se dividió en tres bloques de igual superficie y se pastoreó un bloque por día, al finalizar se ubicaron en cada bloque seis parcelas de 7 x 9 m, asignándose al azar a cada parcela un tratamiento.

Se evaluaron seis tratamientos en arreglo factorial 3 x 2: tres frecuencias y dos intensidades de pastoreo. Las frecuencias consistieron en pastorear cada 21, 28 y 35 días y las intensidades de forraje residual fueron de 5 a 7 y de 9 a $11 \mathrm{~cm}$. El diseño fue bloques al azar con tres repeticiones. Los pastoreos se realizaron con 75 borregas Suffolk x Dorset, de un peso promedio de $65 \mathrm{~kg}$; para asegurar el forraje residual objetivo, se usó una regla durante el pastoreo de cada unidad experimental para garantizar la altura de forraje residual y una vez terminado, se tomaron veinticinco mediciones por unidad experimental con un plato de $30 \times 30 \mathrm{~cm}$, con una regla graduada en el centro $(\mathrm{cm})$.

\section{Variables}

Rendimiento de forraje. Para calcular tanto el forraje presente como el residual, se cortó en cuadros de $0,5 \times 0,5 \mathrm{~m}$ en cincuenta sitios de la parcela de ovillo, el cual se secó en una estufa de aire forzado a $55^{\circ} \mathrm{C}$ por $48 \mathrm{~h}$ y se pesó; con esos datos se generó la siguiente ecuación de regresión $\mathrm{Y}=66,3+80,002 \mathrm{x}$, donde $\mathrm{x}=$ altura de la planta $(\mathrm{cm})$. Las veinticinco mediciones de altura de forraje, obtenidos con el plato, se realizaron dos horas antes y dos horas después de cada pastoreo a cada intensidad y frecuencia, se sustituyeron en la ecuación de regresión de acuerdo con el procedimiento descrito por Hernández-Garay et al. (1997a). El rendimiento estacional y anual del forraje en cada tratamiento, se obtuvieron al sumar el forraje cosechado en los pastoreos correspondientes a los meses de cada estación y en todos los meses del año, respectivamente.

Tasa de crecimiento. La tasa de crecimiento estacional (TC) se calculó mediante la fórmula: TC $=$ $\mathrm{RF} / \mathrm{t}$, descrita por Villareal et al. (2014); donde, TC = tasa de crecimiento (kg MS/ha/d); RF= rendimiento de forraje y $\mathrm{t}=$ días transcurridos en cada estación del año.

Composiciones botánica y morfológica. Para determinar tales variables, al inicio del estudio se colocaron al azar dos cuadros fijos de $0,25 \mathrm{~m}^{2}$ por unidad experimental y se mantuvieron durante todo el experimento. En ellos, un día antes de cada pastoreo, se midió al centro con el plato (Hernández-Garay et al., 1997a), y posteriormente se cortó el forraje de los cuadros fijos a 6 o $10 \mathrm{~cm}$ de altura, según la intensidad de pastoreo. Después, el material cosechado en los cuadros fijos se lavó, se depositó en bolsas etiquetadas A mediados de cada estación del año (dependiendo de cada frecuencia de pastoreo), el material cosechado en cada repetición, se separó en pasto ovillo, material muerto, y otras especies. Para la composición morfológica se tomó toda la muestra de pasto ovillo obtenida en la composición botánica, la cual, se separó en hojas (láminas foliares), tallos (pseudotallos y tallos reproductivos), material senescente e inflorescencias. Cada componente se depositó en bolsas previamente etiquetadas y se secaron en una estufa de circulación de aire forzado a $55^{\circ} \mathrm{C}$ por $48 \mathrm{~h}$ y se pesaron.

Densidad de tallos. Esta variable (tallos $/ \mathrm{m}^{2}$ ) se determinó en cinco ocasiones durante el periodo experimental, con la técnica de Mithchell y Glenday (1958). Para ello, al iniciar la investigación (junio 2005), se tomaron cien muestras al azar dentro de las dieciocho parcelas, con un "sacabocados" de $6,5 \mathrm{~cm}$ de diámetro (Hernández-Garay et al., 1997b) y se registró la densidad promedio de tallos (4421 tallos $/ \mathrm{m}^{2}$ ). Posteriormente, el 11 de julio, 12 de octubre y 22 de diciembre de 2005, así como el 26 de febrero y 16 de mayo de 2006, se tomaron veinticinco muestras aleatorias por repetición, en las cuales se registró esta variable.

Registros climatológicos. Los registros mensuales de temperatura (promedio, máxima, mínima y mínimas extremas), precipitación mensual, horas de brillo solar, radiación global, así como el número de heladas por mes durante el periodo de estudio (Cuadro 1), se obtuvieron de una estación meteorológica situada a $400 \mathrm{~m}$ del sitio experimental. La temperatura media anual en el año de estudio fue de $16,3{ }^{\circ} \mathrm{C}$, en tanto que la precipitación anual fue de $668 \mathrm{~mm}$. Se presentaron veintiocho heladas durante otoño e invierno (13 y 15, respectivamente).

\section{Análisis de datos}

Los datos obtenidos de cada variable y en cada tratamiento se organizaron de manera mensual, estacional o anual para su análisis de varianza, conforme a un diseño experimental de bloques al azar en arreglo factorial $2 \times 3$ con tres repeticiones, mediante el procedimiento GLM del paquete estadístico SAS Institute (1999). Las comparaciones 
Cuadro 1. Características climáticas presentadas durante el periodo de estudio (junio 2005 a junio 2006), en el Campo Experimental Montecillo del Colegio de Posgraduados. Texcoco, Estado de México.

\begin{tabular}{|c|c|c|c|c|c|c|c|c|c|}
\hline \multirow[t]{2}{*}{ Año } & \multirow[t]{2}{*}{ Mes } & \multirow{2}{*}{$\begin{array}{l}\text { Precipitación } \\
\text { pluvial (mm) }\end{array}$} & \multicolumn{4}{|c|}{ Temperatura } & \multirow{2}{*}{$\begin{array}{l}\text { Horas } \\
\text { luz/d }\end{array}$} & \multirow{2}{*}{$\begin{array}{l}\text { Radiación global } \\
\left.\text { (calorías } \mathbf{c m}^{2} / \mathbf{d}\right)\end{array}$} & \multirow{2}{*}{$\begin{array}{c}\text { No. } \\
\text { heladas }\end{array}$} \\
\hline & & & Máxima & Media & Mínima & $\begin{array}{l}\text { Mínima } \\
\text { extrema }\end{array}$ & & & \\
\hline \multirow[t]{7}{*}{2005} & Jun & 33 & 25,3 & 21,5 & 9,1 & 2,5 & 6,3 & 457 & 0 \\
\hline & Jul & 156 & 24,2 & 19,8 & 9 & 4 & 8,4 & 512 & 0 \\
\hline & Ago & 114 & 24,8 & 19,3 & 8,6 & 4 & 7,5 & 428 & 0 \\
\hline & Sep & 52 & 22 & 17,2 & 5,6 & -3 & 8,0 & 483 & 0 \\
\hline & Oct & 54 & 23,2 & 16,4 & 3,4 & -8 & 7,1 & 430 & 0 \\
\hline & Nov & 30 & 20,5 & 13,8 & $-1,4$ & -9 & 7,5 & 411 & 3 \\
\hline & Dic & 10 & 22,1 & 12 & $-3,5$ & -10 & 9,0 & 425 & 10 \\
\hline \multirow[t]{6}{*}{2006} & Ene & 0 & 19,3 & 12,3 & $-3,9$ & -12 & 7,0 & 398 & 11 \\
\hline & Feb & 1 & 25,7 & 14 & $-0,3$ & -9 & 9,0 & 464 & 1 \\
\hline & Mar & 23 & 26,7 & 15,4 & $-0,3$ & $-7,5$ & 9,4 & 495 & 3 \\
\hline & Abr & 32 & 27,9 & 18,3 & 2,7 & -5 & 9,1 & 544 & 0 \\
\hline & May & 83 & 29,8 & 18,5 & 6,2 & 0 & 8,6 & 536 & 0 \\
\hline & Jun & 80 & 26,1 & 18,4 & 5,7 & -2 & 9,0 & 515 & 0 \\
\hline Verano & & 355 & 24 & 19 & 8 & 2 & 8,0 & 474 & 0 \\
\hline Otoño & & 94 & 22 & 14 & -1 & -9 & 7,9 & 422 & 13 \\
\hline Invierno & & 24 & 24 & 14 & -2 & -10 & 8,5 & 452 & 15 \\
\hline \multirow[t]{3}{*}{ Primavera } & & 195 & 28 & 18 & 5 & -2 & 8,9 & 531 & 0 \\
\hline & & 668 & 24,4 & 16,7 & 3,1 & $-4,2$ & 8,1 & 469,0 & 28 \\
\hline & & total & & & & Promedio & & & total \\
\hline
\end{tabular}

de medias entre tratamientos de pastoreo y estaciones del año, se efectuaron mediante la prueba de Tukey con una probabilidad de 0,05 ( $>>0,05)$ ajustada con el procedimiento LSMEANS de SAS.

\section{RESULTADOS Y DISCUSIÓN}

\section{Rendimiento de forraje anual y por estación}

No se registró efecto $(\mathrm{P}>0,05)$ de la interacción ni de la intensidad de pastoreo en el rendimiento de forraje anual y por estación (Cuadro 2). Solo la frecuencia de pastoreo mostró efecto $(\mathrm{P}<0,05)$ en el rendimiento de forraje anual, y el obtenido en primavera y otoño. Los mayores rendimientos de forraje se obtuvieron al pastorear cada 35 días, mostrándose un rendimiento anual de $11700 \mathrm{~kg} \mathrm{MS} / \mathrm{ha}, 14$ y 30\% más forraje que el pastoreo a 21 y 28 días, respectivamente; en el otoño, la superioridad fue de $27 \%$ y más del doble; y en primavera, fue de 30 y $62 \%$.

Al considerar la distribución estacional del rendimiento de forraje, independientemente de la frecuencia e intensidad de pastoreo, el rendimiento estacional se distribuyó de la siguiente forma: 49,5, $31,6,14,1$ y $4,8 \%$ para primavera, verano, invierno y otoño, respectivamente. Las respuestas de las diferentes especies forrajeras al manejo, han sido atribuidas a diferencias en crecimiento, debidas a cambios en temperatura y factores estacionales (Grant et al., 1983). En primavera y verano se registraron las mayores temperaturas y radiación 
Cuadro 2. Rendimiento anual y estacional de pasto ovillo (kg MS/ha) en respuesta a dos intensidades y tres frecuencias de pastoreo de junio 2005 a junio 2006. Campo Experimental Montecillo del Colegio de Posgraduados. Texcoco, Estado de México.

\begin{tabular}{|c|c|c|c|c|c|c|}
\hline \multirow{2}{*}{$\begin{array}{l}\text { Intensidad de } \\
\text { pastoreo }(\mathrm{cm})\end{array}$} & \multirow{2}{*}{$\begin{array}{l}\text { Frecuencia entre } \\
\text { pastoreos (días) }\end{array}$} & \multicolumn{2}{|c|}{2005} & \multicolumn{2}{|c|}{2006} & \multirow[t]{2}{*}{ Anual } \\
\hline & & Verano & Otoño & Invierno & Primavera & \\
\hline \multirow{4}{*}{5 a 7} & 21 & $3242 B$ & $407 \mathrm{abD}$ & $1200 \mathrm{C}$ & $4672 \mathrm{abA}$ & 9521 \\
\hline & 28 & 2995 B & 297 bD & $1825 \mathrm{C}$ & $3727 \mathrm{bA}$ & 8844 \\
\hline & 35 & $3738 \mathrm{~B}$ & $666 \mathrm{abD}$ & $1333 \mathrm{C}$ & $5860 \mathrm{aA}$ & 11598 \\
\hline & Promedio 5 a 7 & $3325 \mathrm{~B}$ & $457 \mathrm{D}$ & $1453 \mathrm{C}$ & $4753 \mathrm{~A}$ & 9987 \\
\hline \multirow{11}{*}{9 a 11} & 21 & 3791B & $608 \mathrm{abD}$ & $1606 \mathrm{C}$ & $4947 \mathrm{abA}$ & 10951 \\
\hline & 28 & 2118B & $285 \mathrm{bD}$ & $1225 \mathrm{C}$ & $4006 \mathrm{bA}$ & 7634 \\
\hline & 35 & 3203 B & $624 \mathrm{abD}$ & $1297 \mathrm{C}$ & $6677 \mathrm{aA}$ & 11801 \\
\hline & Promedio 9 a 11 & 3037 B & $506 \mathrm{D}$ & $1376 \mathrm{C}$ & $5210 \mathrm{D}$ & 10129 \\
\hline & Promedio 21 & 3517 & $507 \mathrm{a}$ & 1403 & $4809 \mathrm{ab}$ & 10236 \\
\hline & Promedio 28 & 2557 & $291 b$ & 1525 & $3866 b$ & 8239 \\
\hline & Promedio 35 & 3470 & $645 \mathrm{a}$ & 1315 & $6269 a$ & 11699 \\
\hline & DMSh & 2330 & 425 & 1874 & 3347 & 6334 \\
\hline & Intensidad (I) & NS & NS & NS & NS & NS \\
\hline & Frecuencia (F) & NS & $*$ & NS & $*$ & $*$ \\
\hline & Interacción F x I & NS & NS & NS & NS & NS \\
\hline
\end{tabular}

$* \mathrm{P}<0,05$; NS $=$ no hay diferencias significativas $(\mathrm{P}>0,05)$. Medias con letras iguales mayúsculas en hileras $\mathrm{y}$ minúsculas en columnas no son estadísticamente diferentes. DMSh = Diferencia Mínima Significativa honesta. F x I $=$ Frecuencia por intensidad.

solar, sin limitaciones de agua, lo que favoreció el crecimiento y rendimiento del pasto ovillo. El $81 \%$ del rendimiento anual registrado durante primaveraverano concuerda con lo reportado por otros autores en los pastos ballico perenne (Velasco et al., 2005) y alfalfa (Rivas et al., 2005) en el Valle de México. El menor rendimiento observado durante el otoño e invierno, pudo ser el resultado de la poca tolerancia del pasto ovillo a las heladas y bajas temperaturas (Hodgson, 1990; Kemp et al., 1999; Velasco et al., 2001), en donde de acuerdo con Hernández-Garay et al. (1997a) dichas temperaturas causan reducción en el crecimiento y tasa de acumulación de forraje, por influencia directa de una baja tasa de aparición y expansión foliar (Horrocks y Vallentine, 1999), ya que para tener la mejor expresión en el crecimiento del pasto ovillo se requieren temperaturas de 18 a $21^{\circ} \mathrm{C}$. Por otra parte, Velasco et al. (2005) en praderas de ballico perenne registraron un comportamiento marcadamente estacional en el rendimiento, siendo los meses de verano e invierno los que presentaron el mayor y menor rendimiento, respectivamente.

\section{Tasa de crecimiento (TC)}

La tasa de crecimiento estacional obtenida se presenta en el Cuadro 3. No se observó efecto de interacción entre intensidad y frecuencia de pastoreo sobre la TC estacional, durante todo el periodo de estudio $(\mathrm{P}>0,05)$. Tampoco se registró efecto de intensidad de pastoreo $(\mathrm{P}>0,05)$. Sólo se observaron efectos de frecuencia en otoño, primavera y en el 
Cuadro 3. Tasa de crecimiento estacional y promedio anual del pasto ovillo (kg MS/ha/d) en respuesta a dos intensidades y tres frecuencias de pastoreo de junio 2005 a junio 2006. Campo Experimental Montecillo del Colegio de Posgraduados. Texcoco, Estado de México.

\begin{tabular}{|c|c|c|c|c|c|c|}
\hline \multirow{2}{*}{$\begin{array}{l}\text { Intensidad de } \\
\text { pastoreo }(\mathrm{cm})\end{array}$} & \multirow{2}{*}{$\begin{array}{l}\text { Frecuencia entre } \\
\text { pastoreos (días) }\end{array}$} & \multirow{2}{*}{$\begin{array}{c}2005 \\
\text { Verano }\end{array}$} & \multicolumn{3}{|c|}{2006} & \multirow[t]{2}{*}{ Promedio } \\
\hline & & & Otoño & Invierno & Primavera & \\
\hline \multirow{4}{*}{5 a 7} & 21 & $37 \mathrm{~B}$ & $4 \mathrm{abD}$ & $15 \mathrm{C}$ & $54 \mathrm{abA}$ & $28 \mathrm{ab}$ \\
\hline & 28 & $33 \mathrm{~B}$ & $3 \mathrm{bD}$ & $20 \mathrm{C}$ & $41 \mathrm{bA}$ & $24 \mathrm{~b}$ \\
\hline & 35 & $42 \mathrm{~B}$ & $7 \mathrm{abD}$ & $15 \mathrm{C}$ & $65 \mathrm{aA}$ & $32 \mathrm{a}$ \\
\hline & Promedio 5 a 7 & 37 & 5 & 17 & 53 & 28 \\
\hline \multirow{11}{*}{9 a 11} & 21 & $42 \mathrm{~B}$ & $7 \mathrm{abD}$ & $18 \mathrm{aC}$ & $55 \mathrm{abA}$ & $30 \mathrm{ab}$ \\
\hline & 28 & $23 \mathrm{~B}$ & $3 \mathrm{bD}$ & $13 \mathrm{aC}$ & $45 \mathrm{bA}$ & $21 \mathrm{ab}$ \\
\hline & 35 & $36 \mathrm{~B}$ & $7 \mathrm{abD}$ & $14 \mathrm{aC}$ & $74 \mathrm{aA}$ & $33 \mathrm{a}$ \\
\hline & Promedio 9 a 11 & 30 & 6 & 15 & 58 & 28 \\
\hline & Promedio 21 & 39 & 6 & 54 & 29 & 29 \\
\hline & Promedio 28 & 28 & 3 & 43 & 23 & 23 \\
\hline & Promedio 35 & 39 & 7 & 70 & 32 & 32 \\
\hline & DMSh & 25,8 & 4,7 & 20,7 & 37,1 & 17,5 \\
\hline & Intensidad (I) & NS & NS & NS & NS & NS \\
\hline & Frecuencia $(\mathrm{F})$ & NS & $*$ & NS & $*$ & $*$ \\
\hline & Interacción F x I & NS & NS & NS & NS & NS \\
\hline
\end{tabular}

$* \mathrm{P}<0,05 ; \mathrm{NS}=$ no hay diferencias significativas $(\mathrm{P}>0,05)$. Medias con letras iguales mayúsculas en hileras y minúsculas en columnas no son estadísticamente diferentes. DMSh = Diferencia Mínima Significativa honesta. F x I = Frecuencia por intensidad.

promedio anual (Cuadro 3), siendo las frecuencias de 35 días las que presentaron los mayores valores con 7,70 y $32 \mathrm{~kg} \mathrm{MS} / \mathrm{ha} / \mathrm{d}$, respectivamente. La tasa de crecimiento estacional presentó el siguiente orden descendiente: primavera $>$ verano $>$ invierno $>$ otoño $(\mathrm{P}<0,05)$. Al respecto, Velasco et al. (2005) observaron las menores producciones de materia seca y TC en otoño e invierno y coincidió con las menores temperaturas (de hasta $-1,8{ }^{\circ} \mathrm{C}$ ).

Los resultados obtenidos en la presente investigación sobre el efecto de intensidades de pastoreo, difiere con otros estudios que mencionan que el mayor rendimiento de forraje y TC, ocurren con una intensidad de pastoreo ligera (Hodgson, 1990) y con intervalos de pastoreo más prolongados (Hodgson, 1990; Garduño et al., 2009). Dichas investigaciones concluyeron que con pastoreos frecuentes y severos, se reduce la persistencia de la pradera. En la presente investigación se encontró que el pasto ovillo es capaz de responder al pastoreo severo, y sólo en forma estacional, se pueden tener diferencias claras entre frecuencias.

A su vez, Hodgson (1990) y Hernández-Garay et al. (1997a), han reportado que las gramíneas alcanzan las mayores TC durante primavera, producto del aumento en la temperatura; y disminuyen en otoño e invierno, a consecuencia de las bajas temperaturas (Cuadro 1), lo que ayuda a entender la estacionalidad en la producción de forraje a través del año. McKenzie et al. (1999) señalaron que la TC es más sensible a la temperatura que las tasas de fotosíntesis y respiración, porque influye sobre la aparición y expansión de hojas, aparición de tallos y estolones y el crecimiento de raíces. Al respecto, Velasco et al. (2005), en praderas de ballico perenne registraron un comportamiento marcadamente estacional en las TC, siendo los meses de verano e invierno los que presentaron la mayor y menor TC, respectivamente. 


\section{Composición botánica y morfológica}

En ninguna de las cuatro estaciones, la interacción frecuencia por intensidad de pastoreo mostró efecto significativo sobre el aporte de ovillo al rendimiento de forraje. La frecuencia e intensidad solamente determinaron la proporción de ovillo en el forraje cosechado en invierno, siendo el pastoreo cada 21 días a una intensidad ligera la que originó que el aporte de ovillo fuera del $40 \%$, incrementándose considerablemente el porcentaje de otras especies a un $60 \%$. En contraste, la proporción de pasto ovillo durante el invierno fue mayor en la frecuencia de pastoreo de 28 días (85\%) superando en 30 y $26 \%$ a la de 35 y 21 días, respectivamente $(\mathrm{P}<0,05)$. También en el invierno el pastoreo severo fue una estrategia para reducir el porcentaje de otras especies y material muerto, y mantener una mayor presencia de la gramínea, con un promedio de $76 \%$ de ovillo comparado con un $57 \%$ del ligero. La principal especie invasora en invierno fue la malva (Malva sylvestris L.), la cual, al parecer no es afectada por las bajas temperaturas, presentándose más notoriamente en el pastoreo ligero y al incrementar el intervalo entre pastoreos (Figura 1).

Durante la primavera y verano no se registraron diferencias $(\mathrm{P}>0,05)$ entre frecuencias de pastoreo en la proporción de pasto ovillo y material muerto (Figura 1). Todos los tratamientos presentaron un porcentaje superior al $60 \%$ de pasto ovillo. El porcentaje promedio del material muerto fue del 19 y $10 \%$ en primavera y verano, respectivamente. Tanto en primavera como en verano, a excepción del pastoreo severo cada 35 días, en donde la presencia de otras especies fue superior al $10 \%(\mathrm{P}<0,05)$, su contribución al rendimiento fue menor al $4 \%$. En este periodo, las especies anuales contribuyeron al rendimiento mayormente cuando el intervalo entre pastoreos y su severidad aumentaron, como lo han mencionado algunos autores (Hernández-Garay et al., 1997a), al señalar que la composición botánica de
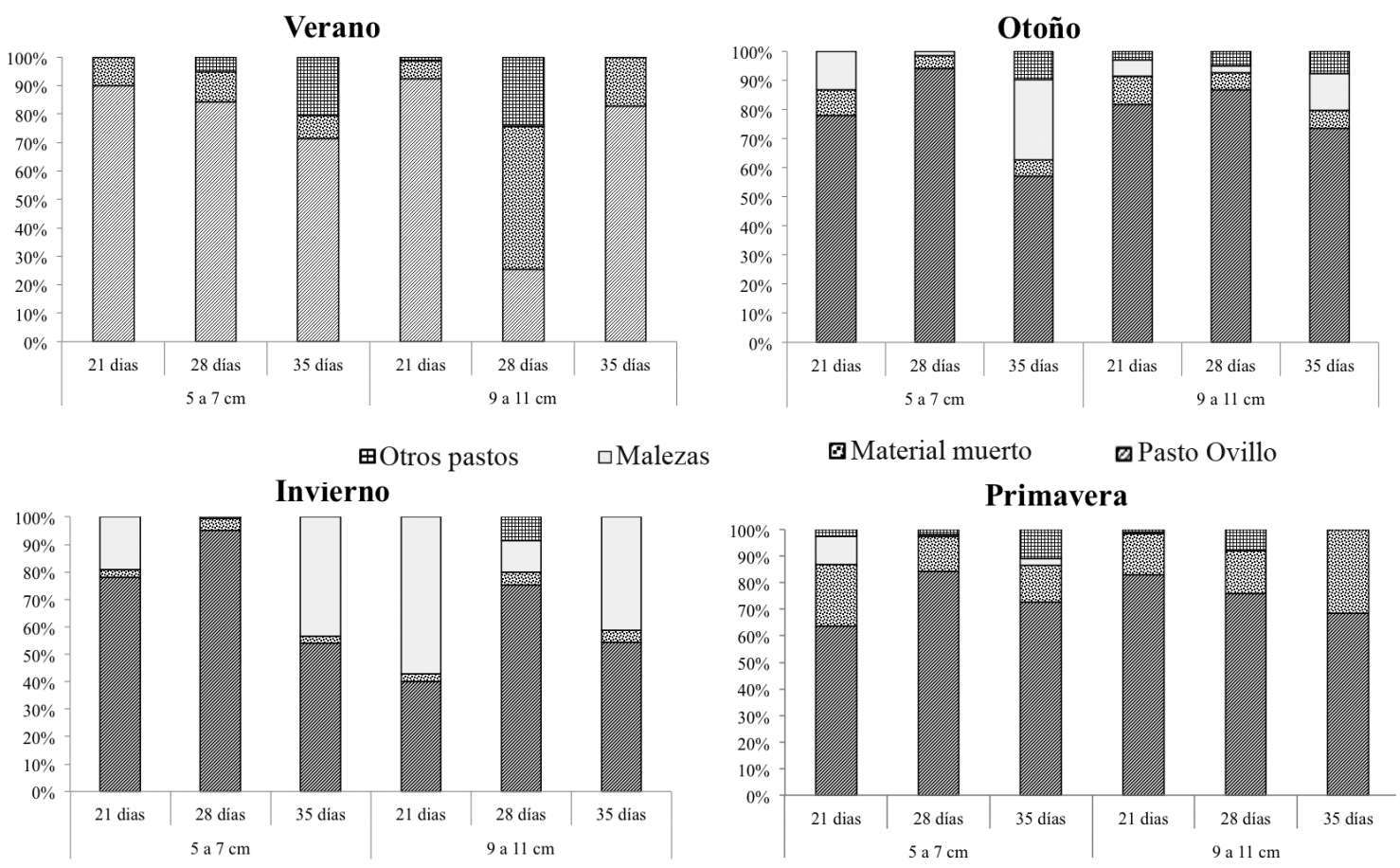

Figura 1. Cambios estacionales en la composición botánica del pasto ovillo en respuesta a dos intensidades y tres frecuencias de pastoreo de junio 2005 a junio 2006. Texcoco, Estado de México. 
las praderas varía con la estación del año y el manejo (Matthew et al., 1999).

Durante el otoño, el menor porcentaje de pasto ovillo se registró al pastorear cada 35 días $(66 \%)$ (Figura 1), siendo 21 y $36 \%$ inferior al de 21 y 28 días $(\mathrm{P}<0,05)$. En contraste, el pastoreo cada 35 días presentó la mayor cantidad de otras especies, con un promedio de $20,5 \%$. En esta estación (otoño), la frecuencia de pastoreo cada 21 días tuvo el mayor porcentaje de material muerto con un promedio de $10,4 \%(\mathrm{P}<0,05)$, seguida de la de 35 y 28 días con 8,4 y $5,4 \%$, respectivamente. En el otoño se incrementó el porcentaje de material muerto, al pastorear más frecuentemente, probablemente debido a que las heladas afectaron más al rebrote joven (Cuadro 1).

Respecto a la contribución de la hoja y el tallo de pasto ovillo al rendimiento, solo se registró efecto de intensidad de pastoreo durante el invierno $(\mathrm{P}<0,05)$ en el porcentaje de hojas, donde el pastoreo severo tuvo los mayores valores (44 vs 40). Durante el verano no se observaron efectos significativos tanto de intensidad como de frecuencia en ninguno de los componentes morfológicos. En otoño, la menor y mayor proporción de hojas y material muerto se presentó al pastorear cada 21 días $(\mathrm{P} \leq 0,05)$. El porcentaje de hojas durante el invierno tendió a disminuir conforme se aumentó el intervalo entre pastoreos de 21 a 35 días $(\mathrm{P} \leq 0,05)$. En contraste, conforme el intervalo entre defoliaciones disminuyó, se redujo el aporte de tallos al rendimiento. Solo se registró efecto de frecuencia en la aportación de hojas al rendimiento durante la primavera, siendo el pastoreo cada 35 días el que mostró los menores valores con $36 \%(\mathrm{P} \leq 0,05)$; esta disminución puede ser explicada por la mayor tasa de recambio de tejido que se presenta en esta estación (Chapman y Lemaire, 1993). Las espiguillas tuvieron un comportamiento similar durante verano y primavera $(\mathrm{P}>0,05)$ y fueron más evidentes en intervalos entre pastoreos más prolongados; similar comportamiento ha sido observado en otros estudios con pasto ovillo y ballico perenne (Velasco et al., 2001 y 2002).

Velasco et al. (2001) reportaron un aumento progresivo en la acumulación de tallo y material muerto conforme se incrementaba la edad de rebrote, similar a lo observado en el presente estudio, en las frecuencias de 21, 28 y 35 días. Al respecto, algunos autores (Hodgson, 1990; Da Silva y Hernández-Garay, 2010) señalan que una vez que la planta intercepta $95 \%$ de la radiación solar, hay un incremento progresivo en el material senescente y muerto, debido al sombreado de las hojas presentes en los estratos inferiores del dosel vegetal, lo cual pudo ocurrir con los intervalos de pastoreo más prolongados.

\section{Densidad de tallos}

No se registró interacción entre intensidad y frecuencia, ni efectos de intensidad o frecuencia de pastoreo $(P>0,05)$, solo se registraron efectos $(P<0,05)$ al comparar los cambios en densidad de tallos a mediados de cada estación (Cuadro 4). En general, se observó que al inicio del estudio (junio de 2005) la densidad promedio fue de 4421 tallos $/ \mathrm{m}^{2}$, lo cual aumentó transcurrido el periodo de evaluación, hasta alcanzar la mayor densidad promedio en diciembre con 8000 tallos $/ \mathrm{m}^{2}(\mathrm{P}<0,05)$; declinando ligeramente en febrero y manteniéndose constante hacia finales de mayo. En praderas de ballico perenne, Velasco et al. (2007) obtuvieron los mayores aumentos en densidad de tallos durante los meses de invierno, con su pico máximo en el mes de febrero. Los valores intermedios registrados en mayo pudieron estar asociados con el inicio de la etapa reproductiva del pasto ovillo (Velasco et al., 2001), durante la cual, los tallos reproductivos tienden a ser más grandes e impiden la penetración de luz solar hacia los estratos inferiores, donde se concentran los tallos recién formados, ocasionándoles la muerte (Hernández-Garay et al., 1997b).

\section{AGRADECIMIENTOS}

Al Consejo Nacional de Ciencia y Tecnología (CONACYT) y a la Línea Prioritaria de Investigación 11 "Sistemas de producción agrícola, pecuaria, forestal, acuícola y pesquera" por el apoyo otorgado para la realización de este trabajo. 
Cuadro 4. Cambios estacionales en la densidad de tallos del pasto ovillo (tallos $/ \mathrm{m}^{2}$ ) en respuesta a dos intensidades y tres frecuencias de pastoreo de junio 2005 a junio 2006. Campo Experimental Montecillo del Colegio de Posgraduados. Texcoco, Estado de México.

\begin{tabular}{|c|c|c|c|c|c|c|}
\hline \multirow{2}{*}{$\begin{array}{l}\text { Intensidad de } \\
\text { pastoreo }(\mathrm{cm})\end{array}$} & \multirow{2}{*}{$\begin{array}{l}\text { Frecuencia entre } \\
\text { pastoreos (días) }\end{array}$} & \multicolumn{3}{|c|}{2005} & \multicolumn{2}{|l|}{2006} \\
\hline & & 11 de julio & 12 de octubre & 22 de diciembre & 26 de febrero & 16 de mayo \\
\hline \multirow[t]{4}{*}{5 a 7} & 21 & $5421 \mathrm{C}$ & $5553 \mathrm{C}$ & $7878 \mathrm{~A}$ & $7207 \mathrm{AB}$ & $7402 \mathrm{~B}$ \\
\hline & 28 & $5421 \mathrm{C}$ & $6101 \mathrm{C}$ & $7109 \mathrm{~A}$ & $6586 \mathrm{AB}$ & $6590 \mathrm{~B}$ \\
\hline & 35 & $5421 \mathrm{C}$ & $5833 \mathrm{C}$ & $7772 \mathrm{~A}$ & $6973 \mathrm{AB}$ & $6424 \mathrm{~B}$ \\
\hline & Promedio 5 a 7 & 5421 & 5829 & 7586 & 6922 & 6806 \\
\hline \multirow[t]{11}{*}{9 a 11} & 21 & $5421 \mathrm{C}$ & $5468 \mathrm{C}$ & $8214 \mathrm{~A}$ & $6730 \mathrm{AB}$ & $6926 \mathrm{~B}$ \\
\hline & 28 & $5421 \mathrm{C}$ & $5906 \mathrm{C}$ & $7810 \mathrm{~A}$ & $7326 \mathrm{AB}$ & $7670 \mathrm{~B}$ \\
\hline & 35 & $5421 \mathrm{C}$ & $7062 \mathrm{C}$ & $9315 \mathrm{~A}$ & $8036 \mathrm{AB}$ & $6420 \mathrm{~B}$ \\
\hline & Promedio 9 a 11 & 5421 & 6145 & 8447 & 7364 & 7005 \\
\hline & Promedio 21 & 5421 & 5510 & 8046 & 6969 & 7164 \\
\hline & Promedio 28 & 5421 & 6003 & 7460 & 6956 & 7130 \\
\hline & Promedio 35 & 5421 & 6448 & 8544 & 7504 & 6422 \\
\hline & DMSh & 0 & 3164 & 3862 & 2524 & 2286 \\
\hline & Intensidad (I) & NS & NS & NS & NS & NS \\
\hline & Frecuencia $(\mathrm{F})$ & NS & NS & NS & NS & NS \\
\hline & Interacción F x I & NS & NS & NS & NS & NS \\
\hline
\end{tabular}

$\mathrm{DMSh}=$ diferencia mínima significativa honesta, $\mathrm{NS}=$ no hay diferencias significativas $(\mathrm{P}>0,05), \mathrm{F}$ x I= frecuencia por intensidad.

Medias con letras iguales mayúsculas en hileras no son estadísticamente diferentes.

\section{LITERATURA CITADA}

Anderson, B., A.G. Matches, y C.J Nelson. 1989. Carbohydrate reserves and tillering of Switchgrass following clipping. Agronomy J. 81:13-16.

Chapman, D.F., y G. Lemaire. 1993. Morphogenetic and structural determinants of plant regrowth after defoliation. N. Z. J. Agric. Res. 26:159-168.

Da Silva, S.C., y A. Hernández-Garay. 2010. Manejo del pastoreo en praderas tropicales. En: Z.M.E.Velazco, A. Hernández-Garay, G.R.A. Perzgrovas, y M.B. Sánchez, editores, Los forrajes y su impacto en el trópico. Universidad Autónoma de Chiapas, Chiapas, México. p. 63-95.

García, E. 1988. Modificaciones al sistema de clasificación climática de Köppen. $4^{\text {a }}$ ed. Universidad Nacional Autónoma de México. México, D. F.
Garduño, V.S., P.J. Pérez, A. Hernández-Garay, H.J.G. Herrera, H.P.A. Martínez, y T.B.N. Joaquín. 2009. Rendimiento y dinámica de crecimiento estacional de ballico perenne, pastoreado con ovinos a diferentes frecuencias e intensidades. Téc. Pec. Méx. 47:189-202.

Grant, A.S., G.T. Barthram, I. Torvell, J. King, y D.A. Elston. 1983. Sward management, lamina turnover and tiller population in continuously stocked Lolium perenne dominated swards. Grass Forage Sci. 38:333-344.

Hernández-Garay, A, C. Matthew, y J. Hodgson. 1997a. Effect of spring grazing management on perennial ryegrass and ryegrass-white clover pastures. 1. Tissue turnover and herbage accumulation. N. Z. J. Agric. Res. 40:25-35.

Hernández-Garay, A., C. Matthew, y J. Hodgson. 1997b. Effect of spring grazing management on perennial ryegrass and ryegrass-white clover pastures. 2 . Tiller 
and growing point densities and population dynamics. N. Z. J. Agric. Res. 40:37-50.

Hernández-Garay, A., C. Matthew, y J. Hodgson. 1999. Tiller size/density compensation in perennial ryegrass miniature swards subject to differing defoliation heights and a proposed productivity index. Grass Forage Sci. 54:347-356.

Hernández-Garay, A, C. Matthew, y J. Hodgson. 2000. The influence of defoliation height on dry matter partitioning and gas exchange of ryegrass miniature swards. Grass Forage Sci. 55:372-376.

Hill, M.J. 1989. The effect of differences in intensity and frequency of defoliation on the grown of siloran Pharalis acuatica and Dactylis glomerata. Aust. J. Agric. Res. 40:333-343.

Hodgson, J. 1990. Grazing management: Science into practice. Longman Scientific and Technical. Harlow, England.

Hodgson, J. 1996. Control del consumo de hierba. En: G.A.N. Martínez, editor, Seminario Internacional TeóricoPráctico: Tópicos selectos en sistemas sustentables de producción animal bajo pastoreo. Facultad de Medicina Veterinaria y Zootecnia, UNAM, México. p. 41-54.

Horrocks, R., y J.F. Vallentine. 1999. Harvested forages. Academic Press, Oval Road, London, UK.

Kemp, C., C. Matthew, y R.J. Lucas. 1999. Pasture species and cultivars. En: J. White, J. Hodgson, editores, New Zealand pasture and crop science. Oxford University Press, Auckland, N.Z. 323 p.

Matthew, C, G. Lemaire, N.R. Sackville, y A. HernándezGaray. 1995. A modified size/density equation to describe size/density relationships for defoliated swards. Ann. of Bot. 76:579-589.

Matthew, C., K.C. Harrington, y J.G. Hampton. 1999. Management of grazing systems. En: J. White, J. Hodgson, editores, New Zealand Pasture and Crop Science. Oxford University Press, Auckland, N.Z. p. 153-174.

Mckenzie, B.A., P.D. Kemp, D.J. Moot, C. Matthew, y R.J. Lucas. 1999. Environmental effects on plant growth and development. En: J. White, y J. Hodgson, editores, New Zealand pasture and crop science. Oxford University Press, Auckland, N. Z. p. 29-44.

Mithchell, K.J., y A.C. Glenday. 1958. The tiller population of pastures. N. Z. J. Agric. Res. 1:305-318.
Ortiz, S.C. 1997. Colección de Monolitos. Depto. Génesis de Suelos. Edafología, IRENAT. Colegio de Postgraduados. Montecillo, Texcoco, Edo. de México. México.

Parsons, A.J., y P. Penning. 1988. The effect of the duration of regrowth on photosynthesis leaf death and the average rate of growth in a rationally grazed sward. Grass Forage Sci. 43:15-27.

Richards, J.H. 1993. Physiology of plants recovering from defoliation. En: M.J. Baker, editor, Proceedings of the 17th International Grassland Congress. SIR Publishing, Wellington, N.Z. p. 85-93.

Rivas, J.M.A., C.C. López, A. Hernández-Garay, y P.J. Pérez. 2005. Efecto de tres regímenes de cosecha en el comportamiento productivo de cinco variedades de alfalfa (Medicago sativa L.). Téc. Pec. Méx. 43:79-92.

SAS Institute. 1999 SAS User's Guide: Statistics. 8 ed. Cary NC, USA. SAS Inst. Inc.

Turner, L.R., D. J. Dornaghy, P.A. Lane, y R.P. Rawnsley. 2006. Effect of defoliation interval on water-soluble carbohydrate and nitrogen reserves, regrowth of leaves and roots, and tiller number of cockfoot (Dactylis glomerata L.) plants. Aust. J. Agric. Res. 57:243-249.

Velasco, Z.M.E., A. Hernández-Garay, H. V. A. González, P. J. Pérez, H. H. Vaquera, y S. A. Galvis. 2001. Curva de crecimiento y acumulación estacional del pasto ovillo (Dactylis glomerata L.). Téc. Pec. Méx. 39:1-14.

Velasco, Z.M.E., A. Hernández-Garay, y H.V.A González. 2002. Curvas estacionales de crecimiento del ballico perenne. Rev. Fitotec. Mex. 25:97-106.

Velasco, Z.M.E., A. Hernández-Garay, y H.V.A. González. 2005. Rendimiento y valor nutritivo del Ballico Perenne (Lolium perenne L.) en respuesta a la frecuencia de corte. Téc. Pec. Méx. 43:247-258.

Velasco, Z.M.E., A. Hernández-Garay, y H.V.A González. 2007. Cambios en componentes del rendimiento de una pradera de ballico perenne, en respuesta a la frecuencia de corte. Rev. Fitotec. Mex. 30:79-87.

Villarreal, G.J.A., G.A. Hernández, H.P.A. Martínez, R.J de D. Guerrero, y Z.M.E Velasco. 2014. Rendimiento y calidad de forraje del pasto ovillo (Dactylis glomerata L.) al variar la frecuencia e intensidad de pastoreo. Rev. Mex. Cienc. Pec. 5:231-245. 